University of Wollongong

Research Online

Australian Institute for Innovative Materials -

Papers

Australian Institute for Innovative Materials

$1-1-2019$

Engineering the poly(vinyl alcohol)-polyaniline colloids for high performance waterborne alkyd anticorrosion coating

Rui Guo

Shaanxi University of Science and Technology

Jiao Wang

Shaanxi University of Science and Technology

Haihua Wang

Northwestern University

Guiqiang Fei

Shaanxi University of Science and Technology

Caiyun Wang

University of Wollongong, caiyun@uow.edu.au

See next page for additional authors

Follow this and additional works at: https://ro.uow.edu.au/aiimpapers

Part of the Engineering Commons, and the Physical Sciences and Mathematics Commons

Research Online is the open access institutional repository for the University of Wollongong. For further information contact the UOW Library: research-pubs@uow.edu.au 


\title{
Engineering the poly(vinyl alcohol)-polyaniline colloids for high performance waterborne alkyd anticorrosion coating
}

\author{
Abstract \\ The ecofriendly waterborne alkyd coatings generally compromise the coating performance. Incorporation \\ of polyaniline into alkyd to form nanocomposites can improve the corrosion resistance; however, the \\ uniform distribution of polyaniline (PANI) within the alkyd resin remains a challenge. In this work, PANI is \\ grafted onto epoxy functionalized phosphorylated poly (vinyl alcohol) (PPVA) with different side-chain \\ configurations in order to improve compatibility and dispersion stability. These functionalized PPVA are \\ synthesized using epichlorohydrin (ECIP) or $\gamma$-glycidyl methacrylate (GMA) as modifying agents, and \\ respectively labeled as E-PPVA and G-PPVA. G-PPVA/PANI dispersion is stable even at a high PANI \\ loading of $50 \%$, while the maximum PANI loading for E-PPVA/PANI is only $30 \%$. With the incorporation of \\ G-PPVA/PANI into waterborne alkyd resins (AR), the coating resistance is increased by 7 orders of \\ magnitude, and the corrosion inhibition efficiency is up to $99.9 \%$. The coating with the incorporation of G- \\ PPVA/PANI also demonstrates superior long-term corrosion resistance in comparison with that AR/E- \\ PPVA/PANI nanocomposite coating.

\section{Disciplines} \\ Engineering | Physical Sciences and Mathematics

\section{Publication Details} \\ Guo, R., Wang, J., Wang, H., Fei, G., Wang, C., Sun, L. \& Wallace, G. G. (2019). Engineering the poly(vinyl \\ alcohol)-polyaniline colloids for high performance waterborne alkyd anticorrosion coating. Applied \\ Surface Science, 481 960-971.

\section{Authors} \\ Rui Guo, Jiao Wang, Haihua Wang, Guiqiang Fei, Caiyun Wang, Liyu Sun, and Gordon G. Wallace
}




\section{Engineering the poly(vinyl alcohol)-polyaniline colloids for high- performance waterborne alkyd anticorrosion coating}

Rui Guo ${ }^{\mathrm{a}}$, Jiao Wang ${ }^{\mathrm{a}}$, Haihua Wang ${ }^{\mathrm{a}, \mathrm{b}, *}$, Guiqiang Fei, Caiyun Wang ${ }^{\mathrm{c}}$, Liyu Sun ${ }^{\mathrm{a}}$, Gordon G. Wallace ${ }^{c}$

${ }^{a}$ Shaanxi Key Laboratory of Chemical Additives for Industry, Shaanxi University of Science and Technology, Xi'an 710021, China

${ }^{\mathrm{b}}$ Department of Material Science and Engineering, Northwestern University, Evanston, Illinois 60208, USA

${ }^{c}$ ARC Centre of Excellence for Electromaterials Science, Intelligent Polymer Research Institute, AIIM Facility, Innovation Campus, University of Wollongong, North Wollongong, NSW 2500, Australia

ABSTRACT: The ecofriendly waterborne alkyd coatings generally compromise the coating performance. Incorporation of polyaniline into alkyd to form nanocomposites can improve the corrosion resistance; however, the uniform distribution of polyaniline (PANI) within the alkyd resin remains a challenge. In this work, PANI is grafted onto epoxy functionalized phosphorylated poly (vinyl alcohol) (PPVA) with different sidechain configurations in order to improve compatibility and dispersion stability. These functionalized PPVA are synthesized using epichlorohydrin (ECIP) or $\gamma$-glycidyl methacrylate (GMA) as modifying agents, and respectively labeled as E-PPVA and GPPVA. G-PPVA/PANI dispersion is stable even at a high PANI loading of 50\%, while the maximum PANI loading for E-PPVA/PANI is only 30\%. With the incorporation of G-PPVA/PANI into waterborne alkyd resins (AR), the coating resistance is increased by 7 orders of magnitude, and the corrosion inhibition efficiency is up to $99.9 \%$. The coating with the incorporation of G-PPVA/PANI also demonstrates superior long-term corrosion resistance in comparison with that AR/E-PPVA/PANI nanocomposite coating. KEYWORDS: Polyaniline, polyvinyl alcohol, alkyd, anti-corrosion, green coating

\section{Introduction}

Corrosion, as a universal phenomenon, has caused an enormous economic loss. Applying an anticorrosion coating is one of the commonly utilized strategies for *Corresponding Authors at: Shaanxi Key Laboratory of Chemical Additives for Ind ustry, Shaanxi University of Science and Technology, Xi' an 710021, China

Haihua Wang: 0000-0002-3045-0008,

*E-mail:whh@sust.edu.cn. 
corrosion protection of metal [1]. However, traditional solventborne coating is harmful to the environment owing to the presence of volatile organic compounds (VOC) [2]. Additionally, fossil fuel-based coatings are expected to be replaced by those bio-based coatings with a reduced environmental footprint. Waterborne bio-based alkyd resins (AR) derived from vegetable oleic acid have attracted extensive attention due to their low cost, low VOC, nontoxicity, non-flammability, good flow and leveling, gloss and adhesion [3-7]. However, the corrosion protection will be weakened when the physical barrier has defects. Moreover, alkyd resins suffer from limited hydrolytic stability, alkali resistance and long drying time [8]. Therefore, various approaches have been put forward to improve the corrosion resistance of alkyd coatings, such as introducing inorganic pigments or anticorrosive additives [4]. Incorporating various nano-materials in the alkyd coatings has been proven to be an effective approach. The used nanomaterials include pigments [9], mesoporous silica nanocontainers [10], carbon nanotubes [11-12], nanoclay [4], M-porphyrin particles [13] and polyaniline (PANI) [14-16]. Pathan et al [17] synthesized butylated melamine fomaldehyde (BMF) modified waterborne soy alkyd. Nano-materials, such as nano- $\mathrm{ZnO}$ and nano- $\mathrm{Fe}_{2} \mathrm{O}_{3}$, are also introduced into waterborne alkyd resin to form nanocomposite coating and improve the anti-corrosion property $[18,19]$. Rahman et al synthesized hyperbranched soya alkyd nanocomposite coating which exhibited superior corrosion resistance performance (impedance $=10^{7} \Omega$ ) [7].

PANI, as a promising anticorrosion additive candidate, not only acts as a physical barrier, but also provides electrochemical anodic and cathodic protection [20-22]. Heretofore, most researches have been mainly focused on the modification of solventborne alkyd/PANI anticorrosion coating [23-27]. The application of PANI in waterborne polymer matrix is always limited by its infusibility and poor processability. Waterborne epoxy [26, 28], polyacrylate [29] and polyurethane emulsion [30] have been commonly used as the matrix for PANI composite coatings. However, reporting on waterborne alkyd/PANI anticorrosion coating is limited. The compatibility and interaction between waterborne alkyd and PANI still remains the main challenge. To improve the water dispersibility of PANI, various approaches have been applied, including self-doping [31, 32], template polymerization [33-35] and counter-ion induced method [36, 37].

In comparison with direct blending or in situ polymerization of water soluble PANI 
and polymer matrix, building chemical bonds between PANI and PVA is beneficial for increasing the compatibility and dispersion stability [38-40]. Different from the previous reports, partially phosphorylated poly(vinyl alcohol) (PPVA) functionalized with epoxy groups was utilized to modify PANI and chemical bonding was achieved between the epoxy functionalized PPVA and PANI in this study. PPVA exhibits more effective stabilization in polymerization, higher water resistance and mechanical property than PVA [41, 42]. Glycidyl methacrylate (GMA) and epichlorohydrin (ECIP) of different molecular configurations were also grafted onto PPVA backbone to incorporate epoxy groups onto the PPVA chains, and the resultant intermediates were designated as G-PPVA and E-PPVA, respectively. PANI was subsequently chemically grafted onto G-PPVA and E-PPVA, and the effects of side chain configuration on the structure and properties of these materials were comparatively studied. G-PPVA/PANI and E-PPVA/PANI have good compatibility with waterborne alkyd resins, especially G-PPVA/PANI. With the incorporation of G-PPVA/PANI, the impedance modulus and coating resistance of AR/G-PPVA/PANI nanocomposites were significantly improved. AR/G-PPVA/PANI nanocomposite coating was also endowed with better long-term corrosion resistance in comparison with AR/E-PPVA/PANI nanocomposite coating.

\section{Materials and methods}

\subsection{Materials.}

Aniline was supplied by Tianjin Chemical Reagent Factory and purified by double distillation under reduced pressure prior to use. Poly(vinyl alcohol) (PVA: $\mathrm{P}_{\mathrm{n}}, 0588 \pm$ $\left.50 ; M_{w}, 19800-24200\right)$ was obtained from Shanghai Yingjia Industrial Development Co., Ltd. Glycidyl methacrylate (GMA), epichlorohydrin (ECIP), urea, diammonium cerium (IV) nitrate (CAN), ammonium persulfate (APS), hydrochloric acid ( $\mathrm{HCl}$ ), phosphoric acid $\left(\mathrm{H}_{3} \mathrm{PO}_{4}\right)$ and potassium hydroxide $(\mathrm{KOH})$ were supplied by Tianjin Tianli Chemical Reagent Co., Ltd. Nitric acid was purchased from Sichuan Xilong chemical Co. Ltd and used as received. Waterborne alkyd resin was synthesized in our laboratory.

\subsection{Preparation of PPVA.}


Phosphorylated PVA (PPVA) was prepared by the reaction of PVA (20 g), phosphoric acid $(41.5 \mathrm{~g})$ and urea $(1.85 \mathrm{~g})$ in an aqueous media at $50{ }^{\circ} \mathrm{C}$ for $8 \mathrm{~h}$, followed by continuous reaction at $95{ }^{\circ} \mathrm{C}$ for $5 \mathrm{~h}$. Purified PPVA was obtained by precipitation in ethanol and dried in vacuum at $40^{\circ} \mathrm{C}$ for $48 \mathrm{~h}$. The extent of phosphorylation for PPVA was $13.16 \%$.

\subsection{Preparation of E-PPVA/PANI dispersions.}

PPVA (10 g) and $100 \mathrm{~mL}$ distilled water were introduced into a $250 \mathrm{ml}$ three-necked flask and stirred at $80{ }^{\circ} \mathrm{C}$ to obtain PPVA solution, and the $\mathrm{pH}$ value was adjusted to 11 with $\mathrm{KOH}$ solution. Then different amounts of ECIP $(0,0.5,1,1.5,2.0,2.5 \mathrm{~g})$ were added dropwise into the reaction system, and the reaction was prolonged for another 1 $\mathrm{h}$ to obtain ECIP modified PPVA (E-PPVA) solution. Afterwards, aniline was added into the E-PPVA solution while the reaction temperature was kept at $80{ }^{\circ} \mathrm{C}$. After $2 \mathrm{~h}$, the $\mathrm{pH}$ value was adjusted to 2 with $\mathrm{HCl}$ followed by the addition of APS solution. The oxidation polymerization was carried at $5{ }^{\circ} \mathrm{C}$ for $24 \mathrm{~h}$ to obtain E-PPVA/PANI in the EPPVA dispersions with different ECIP content. The low-molecular-weight compounds inside the dispersions were removed by dialysis against $0.2 \mathrm{M} \mathrm{HCl}$.

\subsection{Preparation of G-PPVA/PANI dispersions.}

PPVA (10 g), different amounts of $\operatorname{GMA}(0,0.05,0.10,0.15,0.20,0.25,0.30 \mathrm{~mol} / \mathrm{L})$ and distilled water $(100 \mathrm{ml})$ were added into a three-necked flask and vigorously stirred at $60{ }^{\circ} \mathrm{C}$ to obtain uniform dispersion. Then $40 \mathrm{ml}$ of CAN acidic solution was added dropwise into and the reaction was kept at $60{ }^{\circ} \mathrm{C}$ for $5 \mathrm{~h}$. Afterwards, the reaction temperature was raised to $80{ }^{\circ} \mathrm{C}$, followed by the addition of aniline. The reaction was allowed to proceed for $2 \mathrm{~h}$ and then cooled down to $5^{\circ} \mathrm{C}$. Then, nitric acid was utilized to adjust the $\mathrm{pH}$ value to 2 . The oxidation polymerization was initiated with the addition of APS solution and kept at $5{ }^{\circ} \mathrm{C}$ for $24 \mathrm{~h}$ to prepare G-PPVA/PANI in the G-PPVA dispersions with different GMA content. The low-molecular-weight compounds inside the dispersions were removed by dialysis against $0.2 \mathrm{M} \mathrm{HCl}$. The preparation scheme of E-PPVA/PANI and G-PPVA/PANI dispersions is shown in Figure 1.

\subsection{Preparation of nanocomposite films.}


E-PPVA/PANI and G-PPVA/PANI dispersions were cast on leveled tetrafluoroethylene plates to allow them to dry at room temperature. After peeling off, the nanocomposite films were kept in a desiccator to avoid moisture.

\subsection{Preparation of nanocomposite anticorrosion coatings.}

The dispersion with different contents of GMA, ECIP or aniline content were each added into $10 \mathrm{~g}$ waterborne alkyd resin and stirred magnetically for $30 \mathrm{~min}$ to obtain waterborne nanocomposite coatings. The coatings were applied onto the cleaned medium steel sheets with a bar coater.

\subsection{Characterizations.}

Fourier transform infrared (FTIR) spectra were obtained by a Bruker Vector-22 fourier transform infrared spectrometer at a resolution of $4 \mathrm{~cm}^{-1} .{ }^{1} \mathrm{H}-\mathrm{NMR}$ spectra and ${ }^{13} \mathrm{C}$-NMR spectra were determined by Nuclear Magnetic Resonance Spectrometer (NMR, Bruker Avance $400 \mathrm{MHz}$, Karlsruhe, Germany). D-substituted dimethyl sulfoxide (DMSO) was used as solvent. UV-Vis absorption spectra were recorded in the wavelength range of 300-800 nm using an Agilent Cary100 ultraviolet-visible spectrometer with quartz cuvettes. Particle size of the dispersion was analyzed on a Malvern Mastersizer 2000 particle size analyzer. Morphology was observed on a FEI Tecnai G2 F20 transmission electron microscope (TEM). Rheological behaviors were analyzed in a TA AR2000ex rheometer using DIN concentric cylinders geometry. Surface morphology was characterized by a Hitachi S-4800 scanning electron microscopy (SEM). Thermal analysis was performed using a Netzsch TG-209F1 thermogravimetric analyzer (TGA) under nitrogen atmosphere over the temperature range of $30-550{ }^{\circ} \mathrm{C}$ at a heating rate of $10{ }^{\circ} \mathrm{C} / \mathrm{min}$. Surface resistivity was determined with a four-point van der Pauw method with a Keithley 237 high-voltage source and a Keithley 2010 multimeter. Neutral Salt Spray (NSS) tests were evaluated by using a HJ-YW60 salt spray testing machine (Hengjun instrument equipment co., LTD) based on a standard method (GB/T 1771-2007). Hardness was measured according to GB/T6739-1996. Adhesion strength was tested according to GB/T5210-2006 using a 
digital adhesion tester (PosiTest AT-M50mm). Impact strength was measured according to GB/T1732-93. For each specimen, five replicated samples were tested. Neutral salt spray (NSS) testes were evaluated by HJ-YW60 salt spray testing machine (Hengjun instrument equipment Co., Ltd) based on GB/T 1771-2007.

Electrochemical impedance spectroscopy (EIS) was employed to characterize the corrosion protection performance of the coatings. All EIS measurements were performed on an electrochemical workstation (PARSTATMC, USA) at ambient temperature in a classic one-compartment cell with a three-electrode system. A platinum wire was used as the counter electrode $(\mathrm{CE})$; a saturated calomel electrode (SCE) was used as the reference electrode (RE); and the specimen under study with an exposure area of approximately $1.00 \mathrm{~cm}^{2}$ was used as the working electrode (WE); with testing being carried out in a $3.5 \mathrm{wt} \% \mathrm{NaCl}$ solution as electrolyte. All the EIS spectra were recorded over the frequency range of $10 \mathrm{mHz}$ and $100 \mathrm{kHz}$.

\section{Results and discussion}

\subsection{Fabrication of E-PPVA/PANI and G-PPVA/PANI dispersions.}

ECIP and GMA of different molecular configurations were grafted onto partially phosphorylated PVA to generate epoxy groups on PPVA. Subsequently PANI was chemically grafted onto epoxy functionalized PPVA. This approach was able to significantly improve the stability of waterborne E-PPVA/PANI and G-PPVA/PANI dispersions in comparison to PPVA/PANI dispersion. The images in Figure 1B show that precipitation was observed for PPVA/PANI dispersion only after 5-days storage, while precipitation was observed for E-PPVA/PANI-30\% dispersion after 67-days storage. In contrast, no precipitation was detected for G-PPVA/PANI-50\% after storing for 90 days. The above phenomena certify that G-PPVA is more effective in improving the compatibility and interaction between PVA and PANI. It also proves that G-PPVA exhibits more effective stabilization in the chemical graft polymerization of GPPVA/PANI nanocomposites with higher PANI loading.

TEM images of PPVA/PANI, E-PPVA/PANI-30\% and G-PPVA/PANI-50\% dispersions were also provided (Figure 1B). Particle agglomeration occurred in PPVA/PANI dispersion, resulting in the increase of particle size. With the incorporation 
of ECIP and GMA as "bridge" to connect PPVA and PANI, the colloidal particles become more uniform and are well-dispersed in aqueous solution, i.e., uniform ricelike morphology was observed for G-PPVA/PANI-50\% dispersion.

The particle size and polydispersity index (PDI) of PPVA/PANI-30\% dispersion were found to be $1227 \mathrm{~nm}$ and 0.492 (Figure 2), which dramatically decrease to $240.9 \mathrm{~nm}$ and 0.137 for E-PPVA/PANI-30\% dispersion. It suggests that the incorporation of ECIP was able to improve the compatibility and interaction between PPVA and PANI through building covalent bonds via the reaction of epoxy groups in E-PPVA with amine groups in PANI. With increasing aniline content from $20 \%$ to $50 \%$, the particle size of EPPVA/PANI dispersions increased from $162.1 \mathrm{~nm}$ to $431.7 \mathrm{~nm}$. When ECIP was replaced by GMA, i.e., the epoxy groups were incorporated into PPVA chains through grafting GMA into PPVA chains, the particle size of G-PPVA was $202.4 \mathrm{~nm}$ with PDI of 0.236 . Increasing the aniline content from $40 \%$ to $50 \%$, the particle size increased from $202.4 \mathrm{~nm}$ to $298.2 \mathrm{~nm}$, and gradually increased to $317.6 \mathrm{~nm}$ when the aniline content was $60 \%$. It is also worthy to note that the particle size of G-PPVA/PANI-50\% was much lower than that of E-PPVA/PANI-50\%, further demonstrating the enhanced stabilizing effect of G-PPVA. The molecular structure of GMA consists of a methacrylate group and an epoxy group, longer and flexible side chains were formed when GMA was chemically grafted onto the PPVA backbone (Figure 1A). These long chain repeating units act like an internal plasticizer and lower the forces between PPVA backbones, which is beneficial for increasing the free volume and wrapping polyaniline molecules inside the colloidal particles in the subsequent chemical graft polymerization of aniline with epoxy functionalized PPVA, resulting in enhanced stability. However, the side-chain of E-PPVA is very short, hence the amount of free PANI molecules outside the colloidal particles increased with increasing the aniline content, leading to decreased stability and formation of PANI aggregates. 
A

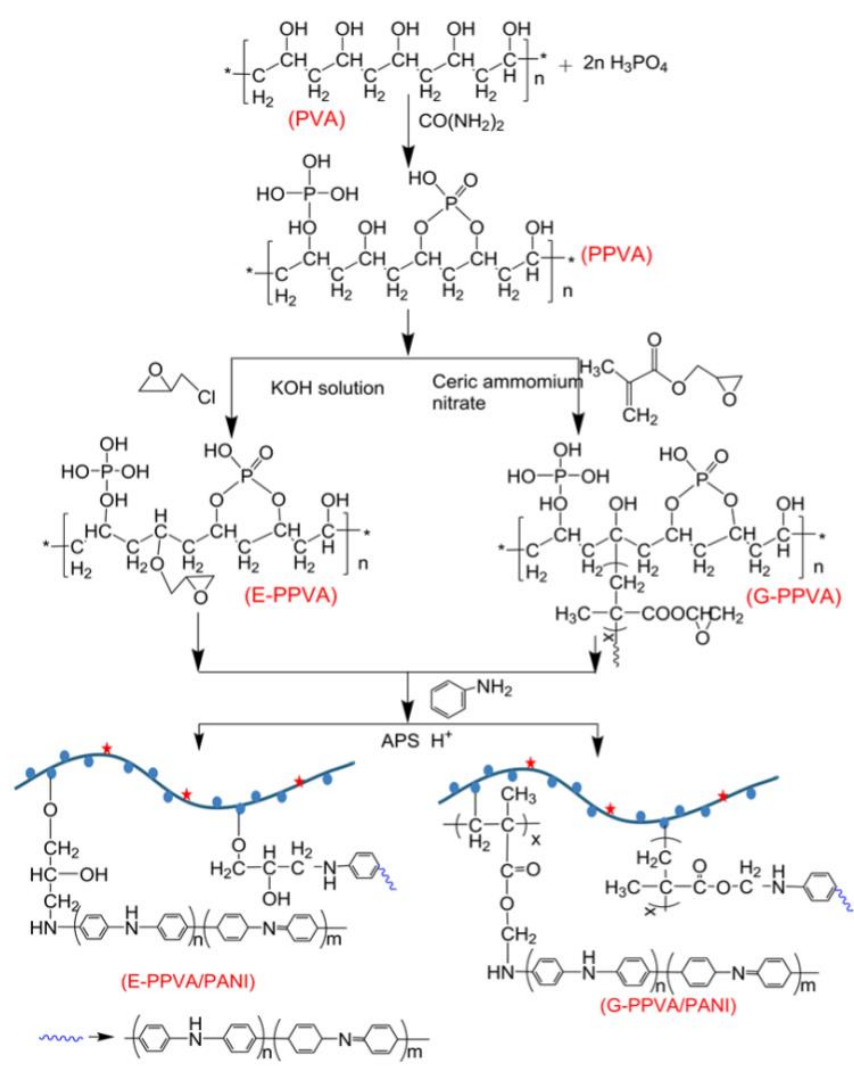

B

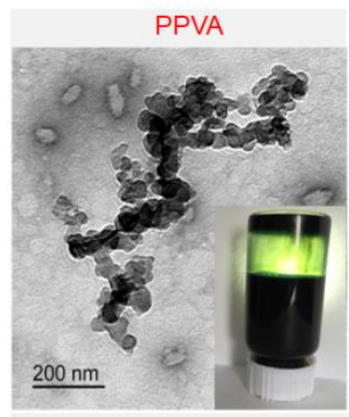

E-PPVA/PANI

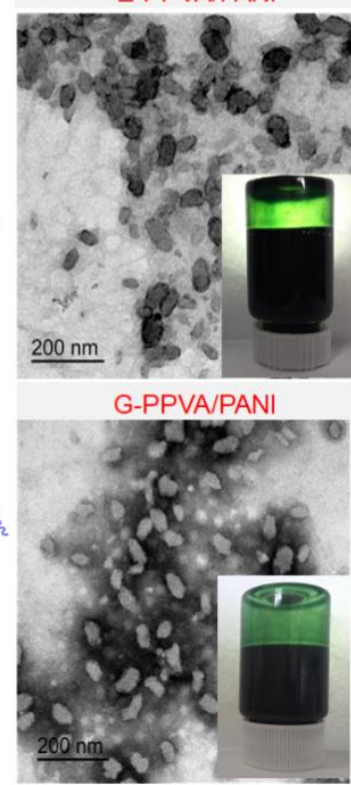

Figure. 1 (A) Schematic preparation of E-PPVA/PANI and G-PPVA/PANI; (B) TEM and optical micrographs of PPVA/PANI, 1.5-E-PPVA/PANI-30\% and 0.2-G-PPVA/PANI-50\% dispersions. Precipitation was observed for PPVA/PANI after 5 days; precipitation was observed for 1.5-EPPVA/PANI-30\% after 67 days; no precipitation was observed for $0.2-$ G-PPVA/PANI-50\% after 90 days.

Rheology is also considered as an efficient tool for exploring structural characteristics and interactions of different materials such as polymer, surfactant, supramolecules in solution, colloid, dispersion or gel [43]. Generally, viscosity is strongly dependent upon the interactions between colloidal particles. The viscosityshear rate curves of E-PPVA/PANI and G-PPVA/PANI dispersions with different ECIP and GMA content are shown in Figure 2E and 2F. It can be observed that the viscosity of E-PPVA/PANI dispersion first increased and then slightly decreased with increasing ECIP content; the viscosity kept decreasing with the increasing shear rate. The increased viscosity indicated the enhanced interaction among E-PPVA/PANI colloidal particles. The particle interaction was gradually decreased with the increasing shear rate. In contrast, the viscosity of G-PPVA/PANI dispersion gradually decreased with the 
increasing GMA content, indicating a weakened particle interaction. The viscosity of G-PPVA/PANI dispersions dramatically decreased and then leveled off when the shear rate was greater than $1 \mathrm{~s}^{-1}$. This suggests that the random arrangement of G-PPVA/PANI colloidal particles in aqueous medium became ordered at higher shear rate.
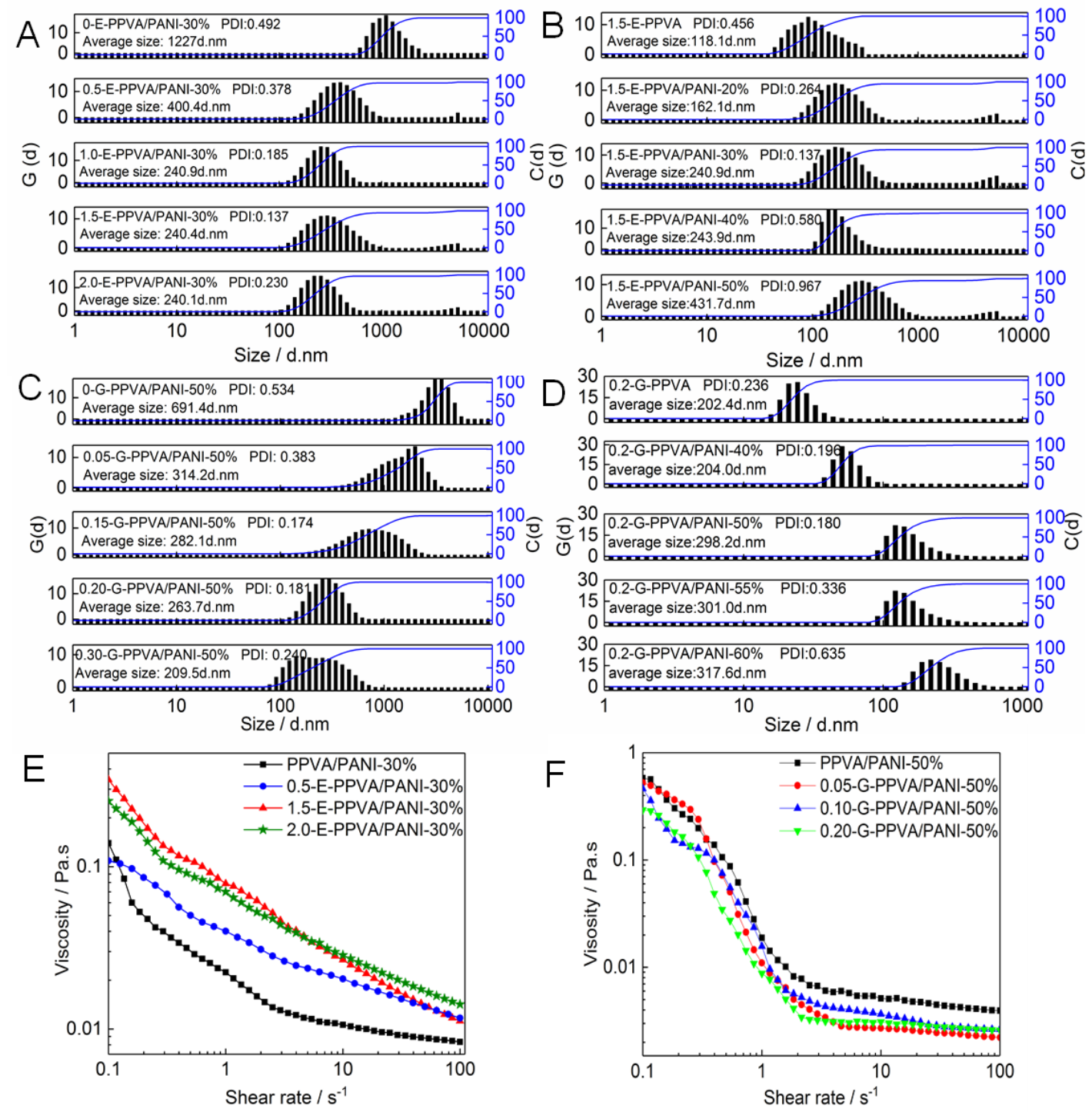

Figure 2. (A) Particle size and distribution of E-PPVA/PANI composites with different ECIP content; (B) Particle size and distribution of E-PPVA/PANI composites with different aniline content; (C) Particle size and distribution of G-PPVA/PANI composites with different GMA content; (D) Particle size and distribution of G-PPVA/PANI composites with different aniline content. (E) Viscosity-shear rate curves of E-PPVA/PANI composites with different ECIP content; (F) Viscosityshear rate curves of G-PPVA/PANI composites with different GMA content.

\subsection{Structure analysis of E-PPVA/PANI and G-PPVA/PANI.}


The structures of PPVA, PPVA/PANI, E-PPVA/PANI and G-PPVA/PANI were characterized by FTIR, ${ }^{1} \mathrm{H}$ NMR and ${ }^{13} \mathrm{C}$ NMR spectra (Figure 3 ). Compared with the FTIR spectrum of PVA, the intensity of $\mathrm{C}=\mathrm{O}$ absorption peak at $1730 \mathrm{~cm}^{-1}$ decreased for PPVA, and a new peak at $1620 \mathrm{~cm}^{-1}$ was detected (Figure 3A), which can be due to the vibration of $\mathrm{P}=\mathrm{O}$ absorption peak [44]. The $\mathrm{P}=\mathrm{O}$ peak shifted to lower wavenumber owing to the hydrogen bond association of the phosphate ester. In the FT-IR spectrum of PPVA/PANI (Figure 3B), the adsorption peaks at $3291 \mathrm{~cm}^{-1}$ is assigned to the overlap of the $-\mathrm{OH}$ groups in PVA and -NH groups in PANI [39]. With the incorporation of PANI into PPVA, the peaks at $1544 \mathrm{~cm}^{-1}$ and $1496 \mathrm{~cm}^{-1}$ in the E-PPVA/PANI spectrum are assigned to skeletal vibrations of the aromatic quinone rings and benzene rings respectively. The peaks at $1415 \mathrm{~cm}^{-1}$ and $1240 \mathrm{~cm}^{-1}$ are attributed to the $\mathrm{C}-\mathrm{N}$ bond stretching vibration in PANI [41]. In comparison with E-PPVA/PANI, the adsorption peak of -NH group in G-PPVA/PANI shifted from $3291 \mathrm{~cm}^{-1}$ to $3216 \mathrm{~cm}^{-1}$ whilst the absorption peak of -OH group shifted from 1721 to $1695 \mathrm{~cm}^{-1}$, indicating the formation of stronger hydrogen bonding for G-PPVA/PANI. The characteristic adsorption peaks of PANI were also observed in the FTIR spectrum of G-PPVA/PANI.
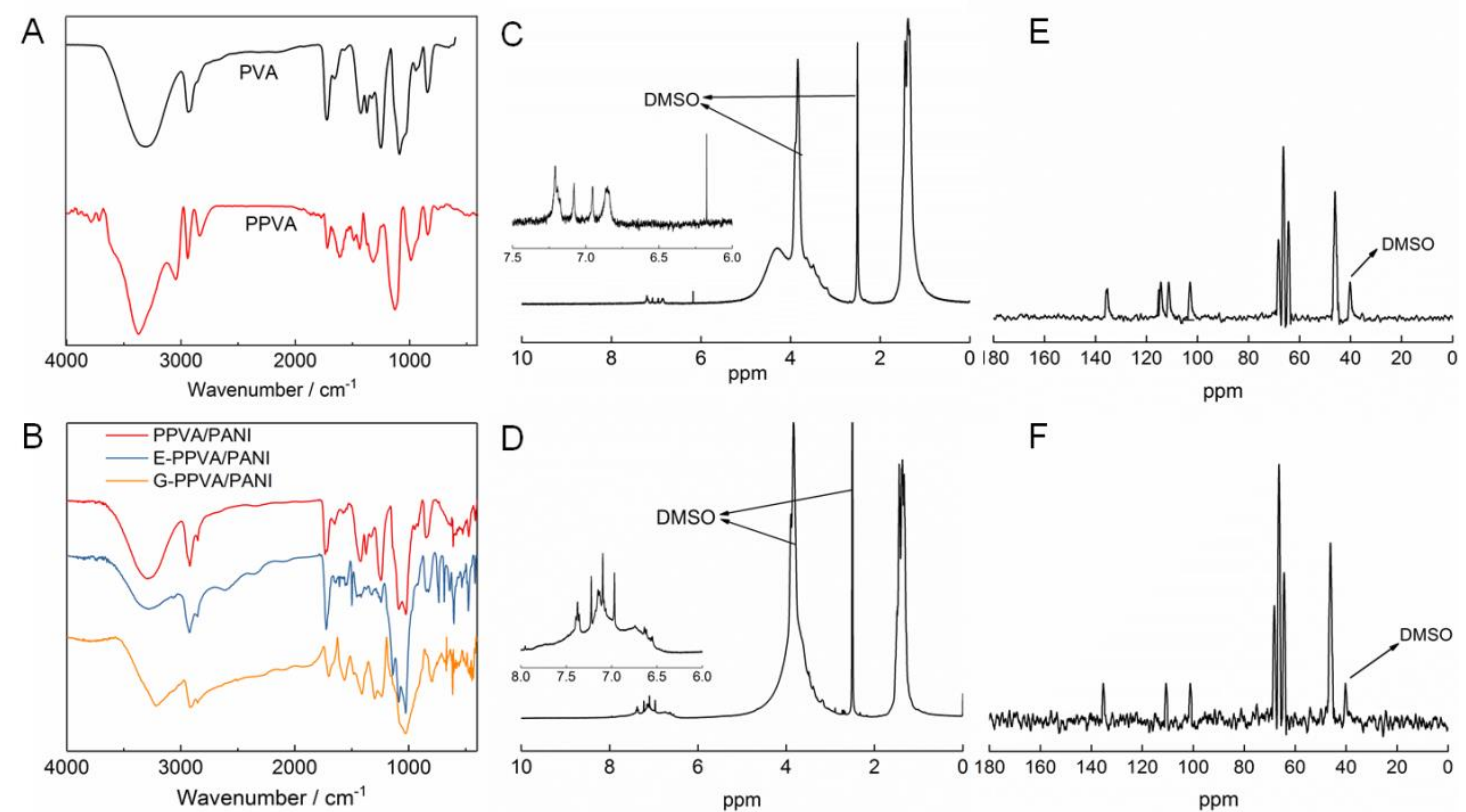

$\mathrm{F}$

Figure 3. FT-IR spectra of PVA and PPVA prepolymers (A); FT-IR spectra of E-PPVA/PANI, PPVA/PANI and G-PPVA/PANI composite films (B); ${ }^{1} \mathrm{H}-\mathrm{NMR}$ spectra of E-PPVA/PANI (C) and G-PPVA/PANI nanocomposites (D); ${ }^{13} \mathrm{C}-\mathrm{NMR}$ spectra of E-PPVA/PANI (E) and G-PPVA/PANI nanocomposites $(\mathrm{F})$.

${ }^{1} \mathrm{H}$ NMR spectra of E-PPVA/PANI and G-PPVA/PANI nanocomposites was shown 
in Figure 3C and 3D. The peaks between 3.5-5.0 ppm were assigned to $\mathrm{C}-\mathrm{H}$ in vinyl acetate units of PVA, and the peaks at 1.1-1.9 ppm were corresponding to $\mathrm{CH}_{2}$ groups in PVA. The peaks at $7.38 \mathrm{ppm}, 7.21 \mathrm{ppm}, 6.86 \mathrm{ppm}$ and $6.25 \mathrm{ppm}$ were correlated to the benzenoid aromatic protons of the PANI backbone [45-47]. It certified the successful incorporation of PANI onto PPVA. In the ${ }^{13} \mathrm{C}$ NMR spectra of E-PPVA/PANI (Figure 3E) and G-PPVA/PANI (Figure 3F) nanocomposites, the peaks at $68.2 \mathrm{ppm}$, $66.3 \mathrm{ppm}$ and $64.5 \mathrm{ppm}$ were attributed to the carbon in PVA chains, the peaks at 135.7 ppm, 115.3 ppm, $103.1 \mathrm{ppm}$ were characteristics of carbon in the PANI chains [48]. It further demonstrated the presence of PANI in the nanocomposites.

Table 1. Elemental analysis and PANI conversion of E-PPVA/PANI and G-PPVA/PANI

\begin{tabular}{|c|c|c|c|c|c|c|c|}
\hline Sample & $\mathrm{N}(\%)^{\mathrm{a}}$ & $\mathrm{N}(\%)^{\mathrm{b}}$ & $\mathrm{C}(\%)^{\mathrm{b}}$ & $\mathrm{H}(\%)^{\mathrm{b}}$ & $\mathrm{O}(\%)^{\mathrm{b}}$ & $\mathrm{P}(\%)^{\mathrm{b}}$ & PANI yield \\
\hline $\begin{array}{l}0.5 \text {-E-PPVA/PANI- } \\
30 \%\end{array}$ & 4.509 & 1.435 & 51.82 & 8.429 & 28.663 & 9.653 & $31.83 \%$ \\
\hline $\begin{array}{l}\text { 1.0-E-PPVA/PANI- } \\
30 \%\end{array}$ & 4.509 & 1.466 & 52.06 & 8.379 & 28.572 & 9.523 & $32.51 \%$ \\
\hline $\begin{array}{l}\text { 1.5-E-PPVA/PANI- } \\
30 \%\end{array}$ & 4.509 & 1.599 & 51.52 & 8.451 & 28.822 & 9.608 & $35.46 \%$ \\
\hline $\begin{array}{l}\text { 2.0-E-PPVA/PANI- } \\
30 \%\end{array}$ & 4.509 & 1.499 & 51.84 & 8.525 & 28.603 & 9.533 & $33.24 \%$ \\
\hline $\begin{array}{l}0.10-G- \\
\text { PPVA/PANI-50\% }\end{array}$ & 7.515 & 2.496 & 51.59 & 8.624 & 28.064 & 9.325 & $33.21 \%$ \\
\hline $\begin{array}{l}0.15-G- \\
\text { PPVA/PANI-50\% }\end{array}$ & 7.515 & 2.606 & 51.50 & 8.506 & 28.041 & 9.347 & $34.68 \%$ \\
\hline $\begin{array}{l}0.20-\mathrm{G}- \\
\text { PPVA/PANI-50\% }\end{array}$ & 7.515 & 3.001 & 51.89 & 8.505 & 27.441 & 9.163 & $39.93 \%$ \\
\hline $\begin{array}{l}0.25-\mathrm{G}- \\
\text { PPVA/PANI-50\% }\end{array}$ & 7.515 & 2.828 & 51.97 & 8.640 & 27.421 & 9.141 & $37.63 \%$ \\
\hline
\end{tabular}

*a represents the theoretical value, $\mathrm{b}$ is the test value.

Furthermore, elemental analysis was performed to investigate the elemental composition in the nanocomposites, as well as the graft efficiency of PANI on PPVA (Table 1). It was found that higher content of PANI was grafted onto G-PPVA in comparison with E-PPVA. The graft efficiency of PANI reached 39.93\% when the GMA content was $0.20 \mathrm{~mol} / \mathrm{L}$.

In the UV-vis spectra (Figure 4), all the sample (E-PPVA/PANI dispersions with different ECIP content and aniline content; G-PPVA/PANI with different GMA content and aniline content) presented three characteristic absorption bands of doped PANI at $300-400 \mathrm{~nm}, 400-500 \mathrm{~nm}$, and $700-800 \mathrm{~nm}$ (Figure 3). The band at 300-400 nm 
corresponds to the $\pi-\pi^{*}$ electron transition of the benzene ring [35]. Band at 400-500 $\mathrm{nm}$ and 700-800 $\mathrm{nm}$ are related to the doping level and exciton transition (polaron/bipolaron transition) generated by the interband charge transfer from benzenoid to quinoid moieties in the protonated PANI [35,39]. The intensity of the absorption bands increased with the ECIP content till reaching the maximum then decreased. As the aniline content increased from $20 \%$ to $40 \%$, intensity of the adsorption band increased first, followed by a decrease at 50\%. Generally, the increased intensity of bands at $400-500 \mathrm{~nm}$ and $700-800 \mathrm{~nm}$ correspond to an increase in conductivity [49]. G-PPVA/PANI dispersions displayed similar variation trends as EPPVA/PANI. However, the intensity of the absorption band at 400-500 nm for GPPVA/PANI was higher than that for E-PPVA/PANI, suggesting an increased conductivity. The band intensity reached a maximum when the aniline content in GPPVA/PANI was $50 \%$, followed by a prominent decline, which may be attributed to the agglomeration of PANI and phase separation.
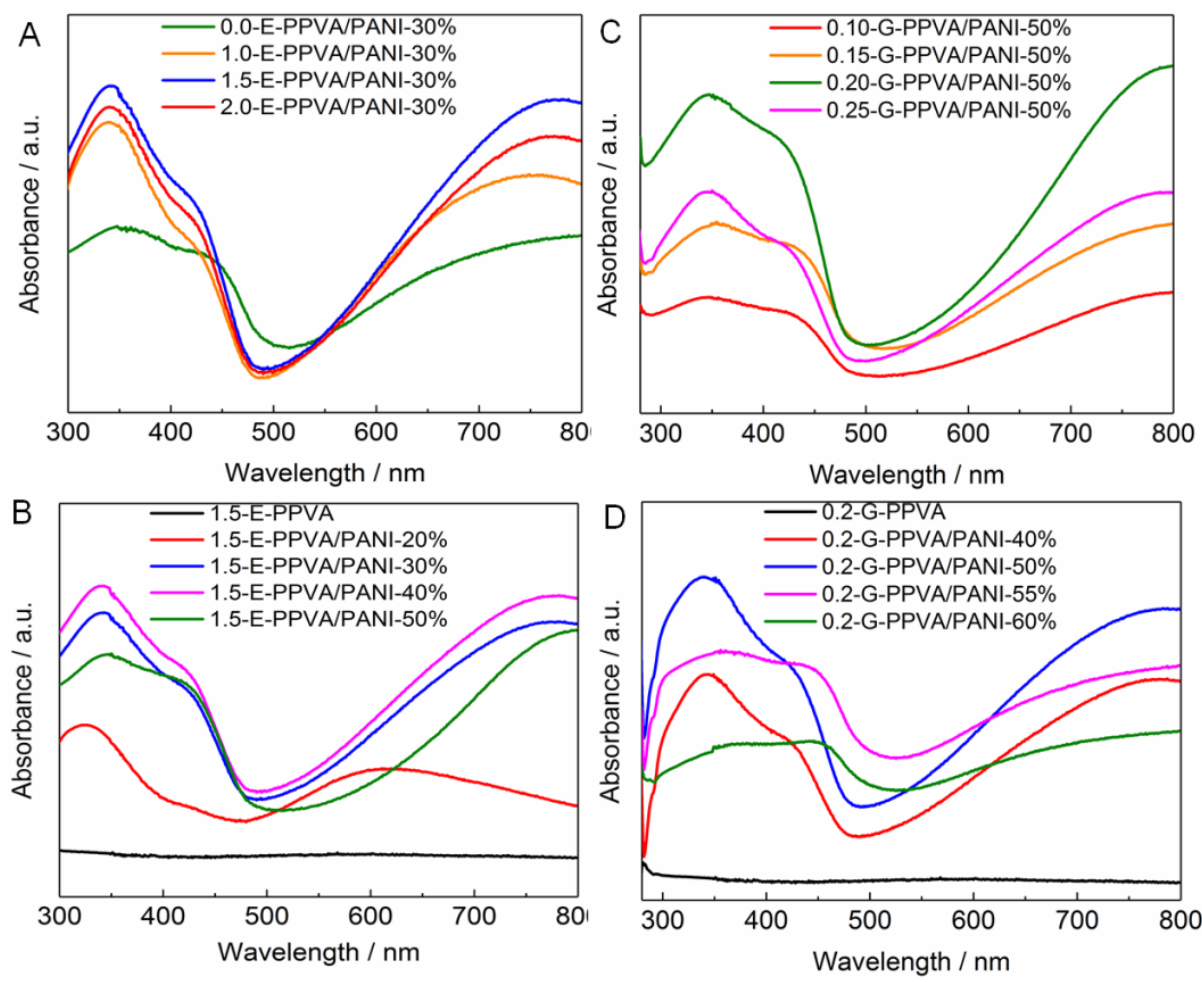

Figure 4. (A) UV-vis spectra of E-PPVA/PANI composites with different ECIP content; (B) UV-vis spectra of E-PPVA/PANI composites with different aniline content; (C) UV-vis spectra of GPPVA/PANI composites with different GMA content; (D) UV-vis spectra of G-PPVA/PANI composites with different aniline content. 


\subsection{Conductivity, morphology, and thermal behavior of nanocomposite films.}

The conductivity of nanocomposite films is presented in Figure 5. It increased when ECIP and GMA molecules were grafted onto the PPVA backbone and introduced as "bridge" to connect PPVA and PANI. The conductivity of G-PPVA/PANI nanoncomposites was higher than that of E-PPVA/PANI, which can be attributed to the homogeneous distribution of PANI in the G-PPVA matrix and formation of connected conductive paths. It reached a maximum when the ECIP content was $1.5 \mathrm{~g}$. The content of GMA affected the conductivity of G-PPVA/PANI nanocomposite as well; it increased to a maximum when the GMA content was $0.20 \mathrm{~mol} / \mathrm{L}$.
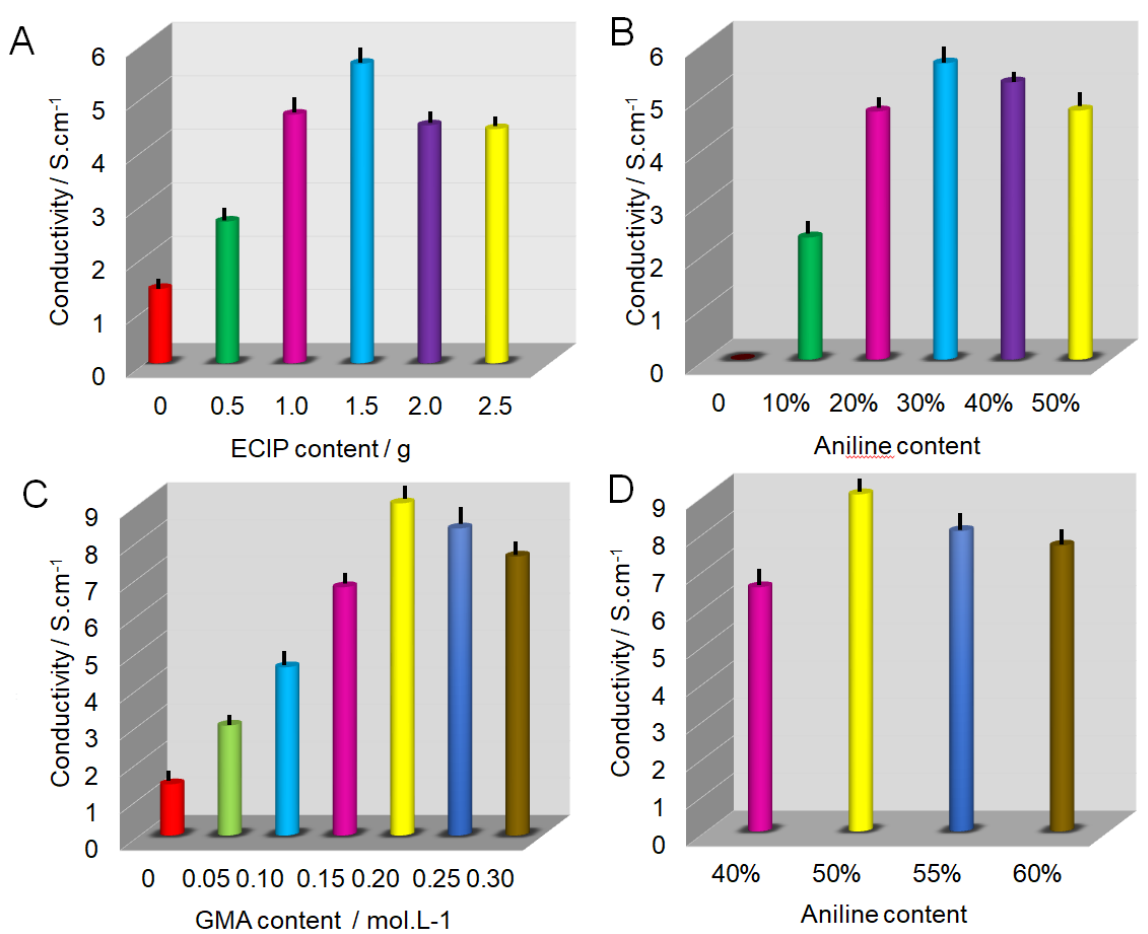

Figure 5. (A) Conductivity of E-PPVA/PANI nanocomposites with different ECIP content; (B) Conductivity of E-PPVA/PANI nanocomposites with different aniline content; (C) Conductivity of G-PPVA/PANI nanoncomposites with different GMA content; (D) Conductivity of G-PPVA/PANI nanocomposites with different aniline content.

SEM images of PPVA/PANI, 1.5-E-PPVA/PANI-30\% and 0.2-G-PPVA/PANI-50\% nanocomposite films were used to characterize the distribution of PANI in the PPVA matrix (Figure 6A). PANI was distributed into PPVA in the form of irregular particles for the PPVA/PANI physical blends. The particle size of PANI in E-PPVA/PANI-30\% film decreased owing to the increased compatibility between PANI and E-PPVA. Notably, the surface of the G-PPVA/PANI-50\% film became much smoother, 
demonstrating that PPVA grafted with GMA chains was more favorable for forming an homogeneous nanocomposite in comparison with the ECIP chains.

TG and DTG curves of 1.5-E-PPVA/PANI-30\% and 0.2-G-PPVA/PANI-50\% are shown in Figure 6B. The 0.2-G-PPVA/PANI-50\% nanocomposite displayed higher thermal stability than 1.5-E-PPVA/PANI-30\%. There were mainly two degradation processes. The first stage $\left(154.9-307.9{ }^{\circ} \mathrm{C}\right.$, peak at $\left.192.9^{\circ} \mathrm{C}\right)$ can be ascribed to the breaking of side-groups; the second stage $\left(329.7-548.1^{\circ} \mathrm{C}\right)$ can be due to the breaking of PPVA and PANI backbones. In contrast, the degradation temperature of 0.2-GPPVA/PANI-50\% shifted to higher temperature indicating an enhanced thermal stability: the first stage appeared at $201.1-316.1^{\circ} \mathrm{C}$ with the peak at $229.5^{\circ} \mathrm{C}$; the second stage was between $331.1-550.0^{\circ} \mathrm{C}$ with the peak at $447.5^{\circ} \mathrm{C}$. The enhanced thermal stability can be attributed to the improved compatibility and interaction between GPPVA and PANI. Based on the above results, the 1.5-E-PPVA/PANI-30\% and 0.2-GPPVA/PANI-50\% dispersions were thereby selected to prepare nanocomposite coatings using waterborne alkyd resin as matrix.

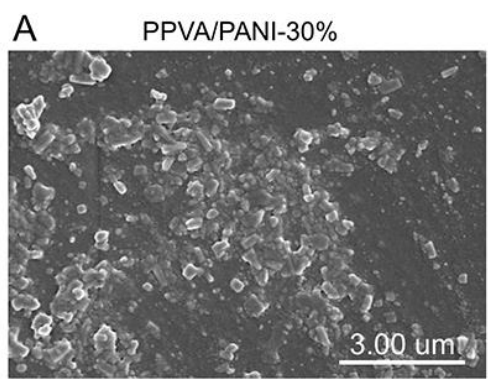

PPVA/PANI-50\%

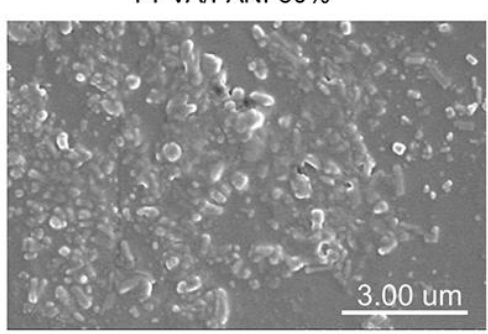

1.5-E-PPVA/PANI-30\%

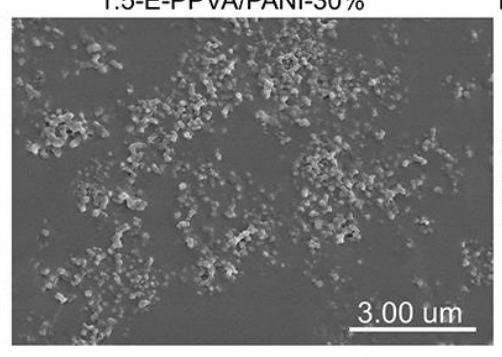

0.2-G-PPVA/PANI-50\%

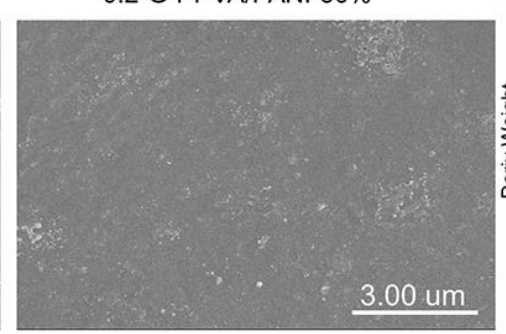

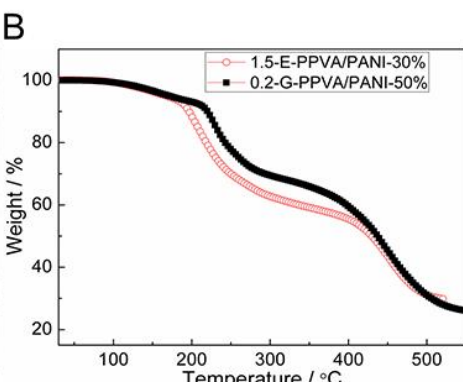

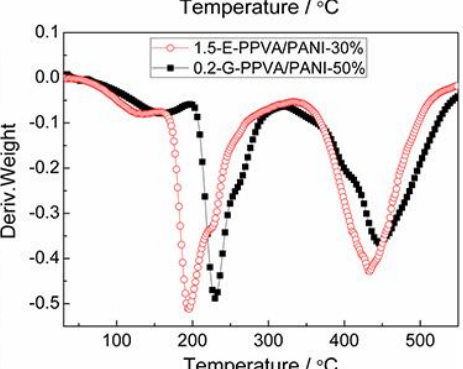

Figure 6. (A) Surface morphology of PPVA/PANI-30\%, 1.5-E-PPVA/PANI-30\%, PPVA/PANI-50\% and 0.2-G-PPVA/PANI-50\% films; (B) TG (upper) and DTG (bottom) curves of 1.5-E-PPVA/PANI and 0.2-G-PPVA/PANI composites.

\subsection{Corrosion resistance of nanocomposite coatings.}

Electrochemical impedance spectroscopy (EIS), as a powerful non-destructive measurement, is capable of obtaining significant quantitative and qualitative data on 
corrosion. EIS Bode plots of pure AR, AR/E-PPVA/PANI and AR/G-PPVA/PANI nanocomposite coatings are shown in Figure 7. The impedance modulus at lower frequency $(|\mathrm{Z}|)$ is generally considered as a measure of corrosion resistance; higher $|\mathrm{Z}|$ value corresponds to lower capacitance and higher resistance of the coatings. As shown in Figure $7 \mathrm{~A}$ and $7 \mathrm{~B},|\mathrm{Z}|$ value at lower frequency for AR was only $6.585 \times 10^{2} \Omega . \mathrm{cm}^{2}$. It increased to $1.751 \times 10^{7} \Omega . \mathrm{cm}^{2}$ with $2.5 \%$ of E-PPVA/PANI, but then significantly decreased to $8.919 \times 10^{5} \Omega . \mathrm{cm}^{2}$ when the E-PPVA/PANI content was $3.5 \%$. The increase of impedance modulus can be due to the passivating effect of E-PPVA/PANI. The decrease of impedance modulus at higher E-PPVA/PANI content may be attributed to the agglomeration and phase separation of E-PPVA/PANI in the AR matrix. $|Z|$ value of AR/G-PPVA/PANI nanocomposite coating increased to $3.173 \times 10^{9} \Omega . \mathrm{cm}^{2}$ when the G-PPVA/PANI content was 3.5\%, which is much higher than that reported in recent literature (impedance $=10^{7} \Omega$ ) [7]. The higher $|\mathrm{Z}|$ value for G-PPVA/PANI with the same nanofiller content indicates that G-PPVA/PANI possessed better compatibility with the AR matrix in comparison with E-PPVA/PANI.
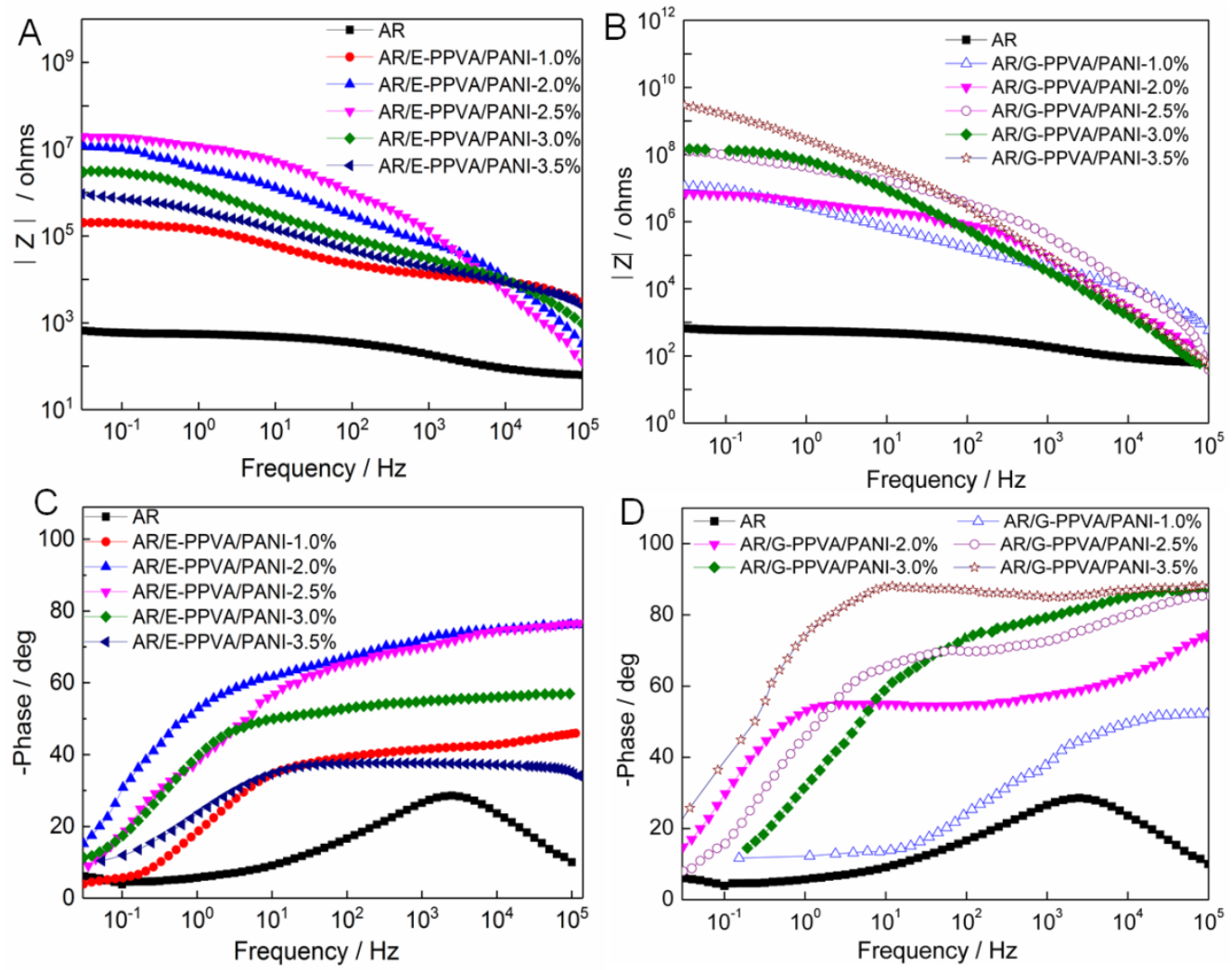

Figure 7. Bode impedance plots of AR/E-PPVA/PANI (A) and AR/G-PPVA/PANI (B) nanocomposite coatings with different PPVA/PANI content. Bode phase plots of AR/E-PPVA/PANI (C) and AR/G-PPVA/PANI (D) nanocomposite coatings with different PPVA/PANI content. 
Bode phase plots of AR, AR/E-PPVA/PANI and AR/G-PPVA/PANI nanocomposite coatings were also obtained to investigate the corrosion process (Figure 7C, 7D). In general, the corrosion process may be divided into two stages. At the first stage, the penetration of electrolyte into the coating leads to the decreased coating resistance (Rc). Then, the electrolyte reached to the coating/metal interface, resulting in metal corrosion. Two-time constant was detected for pure AR coating, suggesting poor corrosion resistance. In contrast, AR/E-PPVA/PANI and AR/G-PPVA/PANI nanocomposite coatings displayed only one-time constant. It was also found that the phase angle at high frequency $\left(10^{5} \mathrm{~Hz}\right)$ of AR/G-PPVA/PANI was higher than that of AR/EPPVA/PANI. This further demonstrates that AR/G-PPVA/PANI was endowed with better anticorrosive performance than AR/E-PPVA/PANI.

A
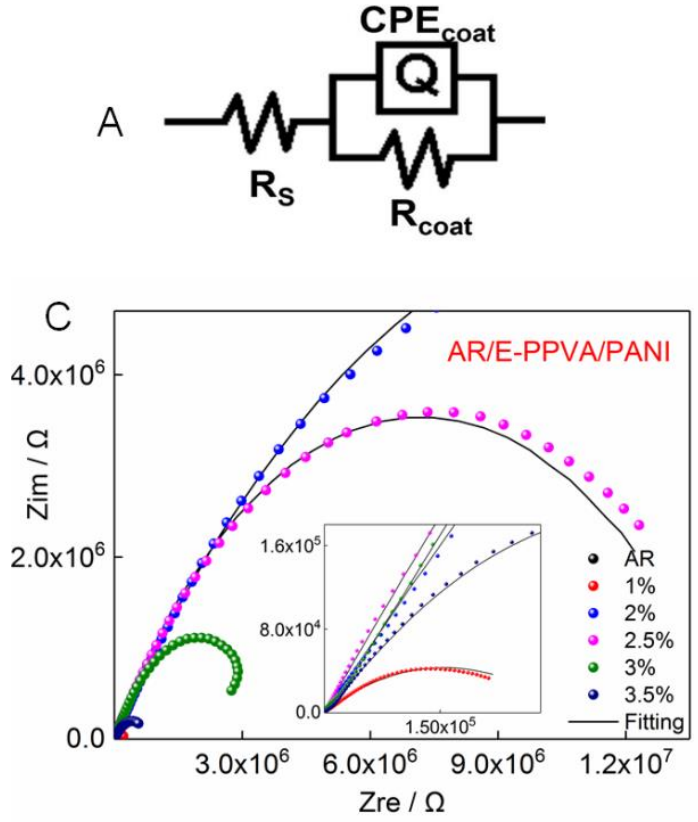
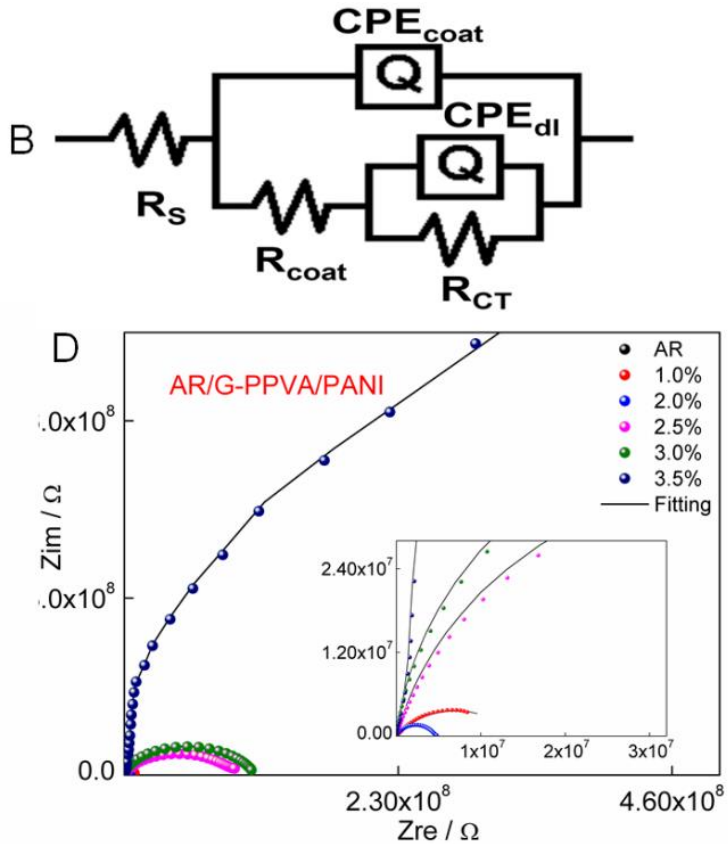

Figure 8. Electrical equivalent circuit models (A and B) for nanocomposite coatings. Nyquist plots for AR/E-PPVA/PANI (C) and AR/G-PPVA/PANI (D) nanocomposite coatings prepared with different PPVA/PANI content.

Nyquist plots of AR/E-PPVA/PANI and AR/G-PPVA/PANI nanocomposite coatings are illustrated in Figure 8. Two equivalent circuit (EEC) models were employed to simulate the impedance data, where $R_{s}$ is solution resistance, $R_{\text {coat }}$ represents the coating resistance (an indicator to estimate the barrier properties of coating), $\mathrm{CPE}_{\text {coat }}$ corresponds to the constant phase element of coating, $\mathrm{R}_{\mathrm{CT}}$ and $\mathrm{CPE}_{\mathrm{dl}}$ represents charge transfer resistance and double-layer constant phase element, respectively. The 
corresponding electrochemical parameters are summarized in Table S1. It can be seen that $\mathrm{R}_{\text {coat }}$ displayed a similar variation trend as the impedance modulus, and the $\mathrm{CPE}_{\text {coat }}$ showed an opposite trend. The $\mathrm{R}_{\text {coat }}$ and capacitive arcs of AR/E-PPVA/PANI were found to be higher than that of pure $A R$ by five orders of magnitude, $R_{\text {coat }}$ and capacitive arcs of AR/G-PPVA/PANI were seven orders higher than that of pure AR. These results demonstrate that G-PPVA/PANI is more effective in improving the corrosion resistance of waterborne AR coating compared with E-PPVA and PANI.
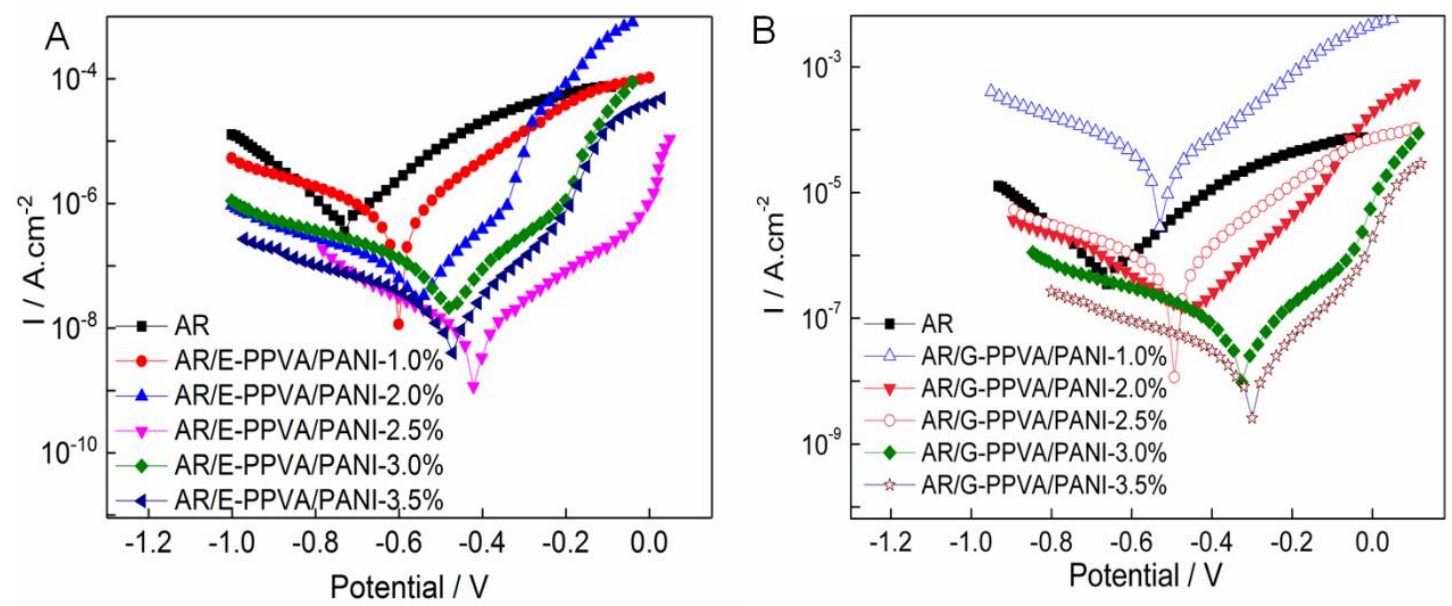

Figure 9. Potentiodynamic polarization curves of AR/E-PPVA/PANI nanocomposites (A) and AR/G-PPVA/PANI nanocomposites (B) with AR as control.

Figure 9 displays the potentiodynamic polarization curves of AR, AR/E-PPVA/PANI and AR/G-PPVA/PANI nanocomposite coatings, where the related parameters including $\mathrm{E}_{\text {corr }}$ (corrosion potential), $\mathrm{I}_{\text {corr }}$ (corrosion current density), $\mathrm{IE}_{(\mathrm{I})}$ (corrosion inhibition efficiency), $\beta_{\mathrm{a}}$ (anodic) and $\beta_{\mathrm{c}}$ (cathodic) Tafel slope are summarized in Table S2. The corrosion inhibition efficiency was calculated based on equation (1) [50].

$$
\mathrm{IE}(\mathrm{I})=\frac{\mathrm{I}_{\text {corr }}^{0}-\mathrm{I}_{\text {corr }}}{\mathrm{I}_{\text {corr }}^{0}} \times 100 \%
$$

Where $\mathrm{I}^{0}$ corr is the corrosion current density of uncoated metal substrate, $\mathrm{I}_{\text {corr }}$ is the corrosion current density of coated metal substrate.

It is found that $\mathrm{I}_{\text {corr }}$ decreased with increasing content of E-PPVA/PANI from $1.0 \%$ to $2.5 \%$, but then increased when the content was increased further to $3.5 \%$. In contrast, $\mathrm{I}_{\text {corr }}$ kept decreasing from $5.81 \times 10^{-6}$ to $1.15 \times 10^{-9} \mathrm{~A} / \mathrm{cm}^{2}$ as the content of G-PPVA/PANI content increased from $1.0 \%$ to $3.5 \%$. Compared with AR/E-PPVA/PANI, $\mathrm{E}_{\text {corr }}$ of AR/G-PPVA/PANI displayed a positive shift (by $92 \mathrm{mV}$ ). In addition, it was a large positive shift (by $404 \mathrm{mV}$ ) compared with $\mathrm{AR}$, and the corresponding $\mathrm{IE}_{(\mathrm{I})}$ increased by 
up to $99.9 \%$. Generally, $\mathrm{I}_{\text {corr }}$ represents the intensity of cathodic oxygen reduction and anodic dissolution of metal ions, a lower $I_{\text {corr }}$ value and a higher $E_{\text {corr }}$ value indicate a lower corrosion dynamic rate. The electrochemical parameters in Table S2 certify that G-PPVA/PANI was much more effective in improving the corrosion resistance of waterborne AR resin compared with E-PPVA/PANI. These results are consistent with EIS measurement results.
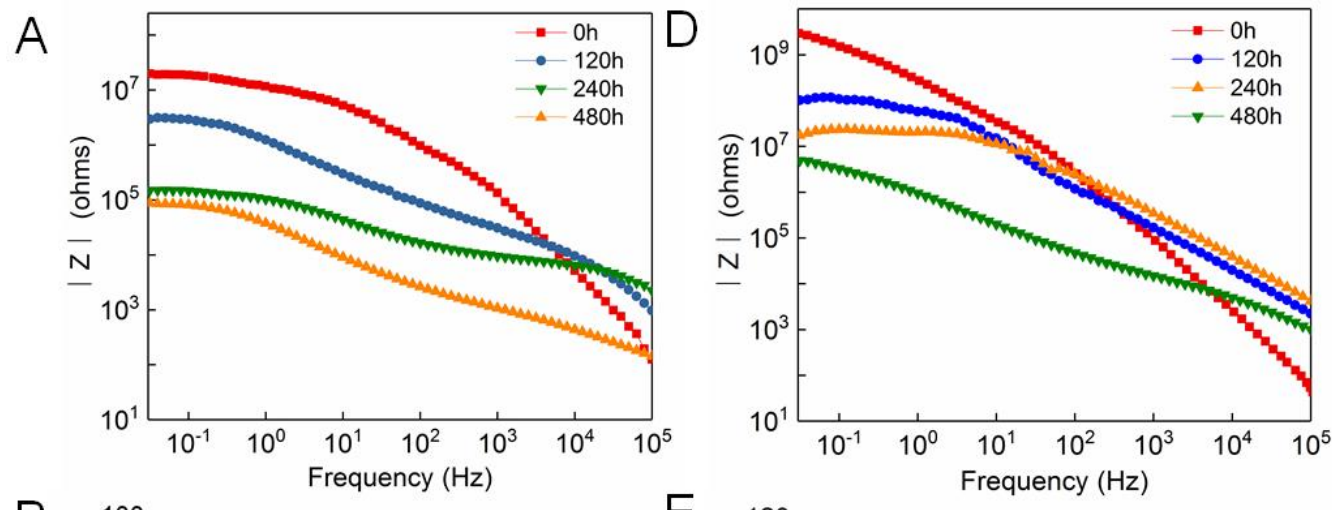

B
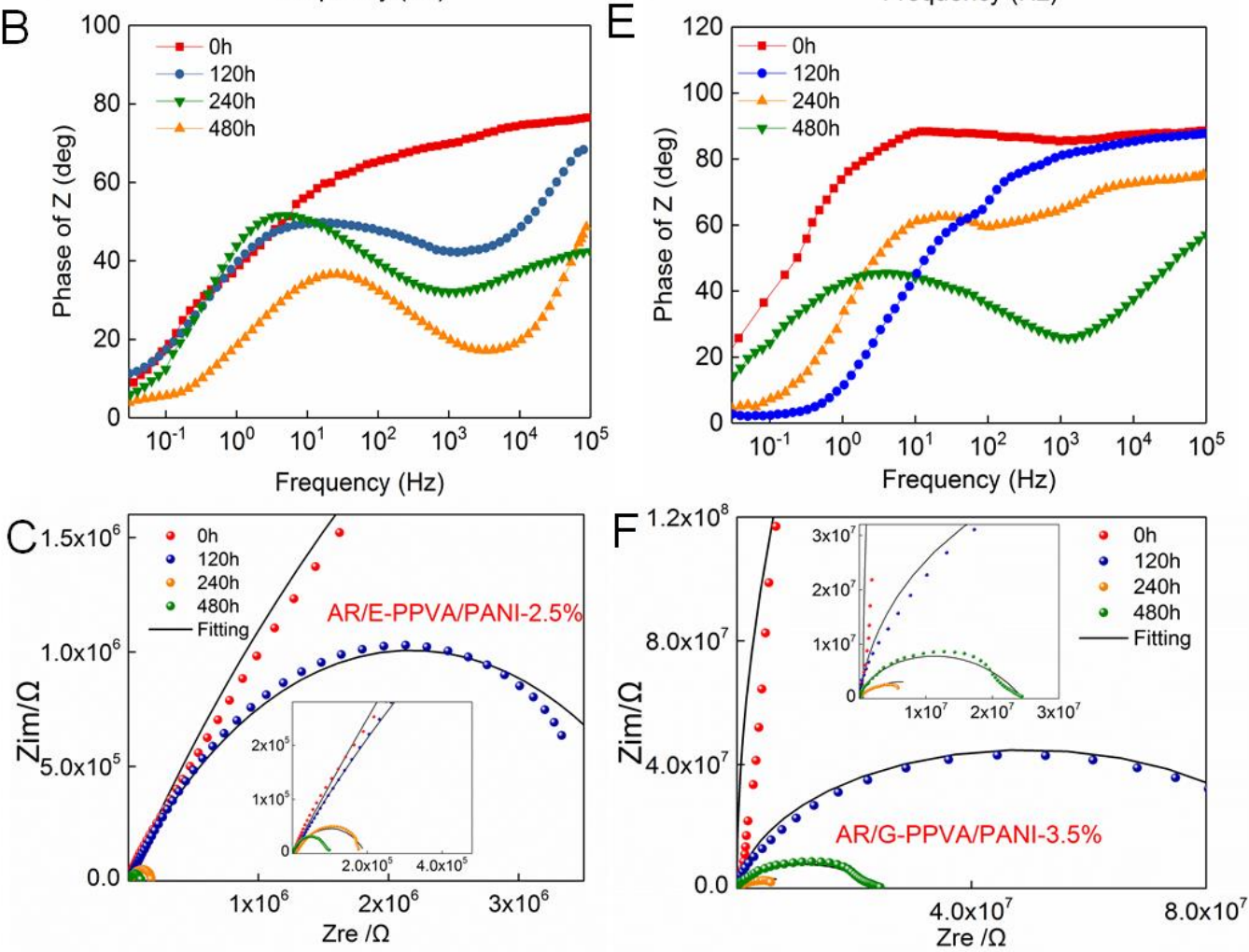

Figure 10. Bode impedance plots (A), Nyquist plots (B) and Bode phase plots (C) for AR/EPPVA/PANI nanocomposite coating at different immersion time; Bode impedance plots (D), Nyquist plots (E) and Bode phase plots (F) for AR/E-PPVA/PANI nanocomposite coating at different immersion period.

EIS was also utilized to investigate the corrosion resistance of AR/E-PPVA/PANI2.5\% and AR/G-PPVA/PANI-3.5\% nanocomposite coatings at different immersion 
periods to investigate the long-term corrosion resistance (Figure 10). The variation of electrochemical parameters with time is illustrated in Table S3. The impedance modulus and coating resistance decreased with the increasing immersion period, indicating weakened corrosion resistance. Two-time constant was detected for AR/E-PPVA/PANI$2.5 \%$ after immersion of $120 \mathrm{~h}$, while two-time constant was observed for AR/GPPVA/PANI-3.5\% after immersion of $480 \mathrm{~h}$. The increased impedance modulus, together with higher $\mathrm{R}_{\text {coat }}$ and $\mathrm{CPE}_{\text {coat }}$ further demonstrate that AR/G-PPVA/PANI-3.5\% nanocomposite coating is endowed with better long-term resistance.

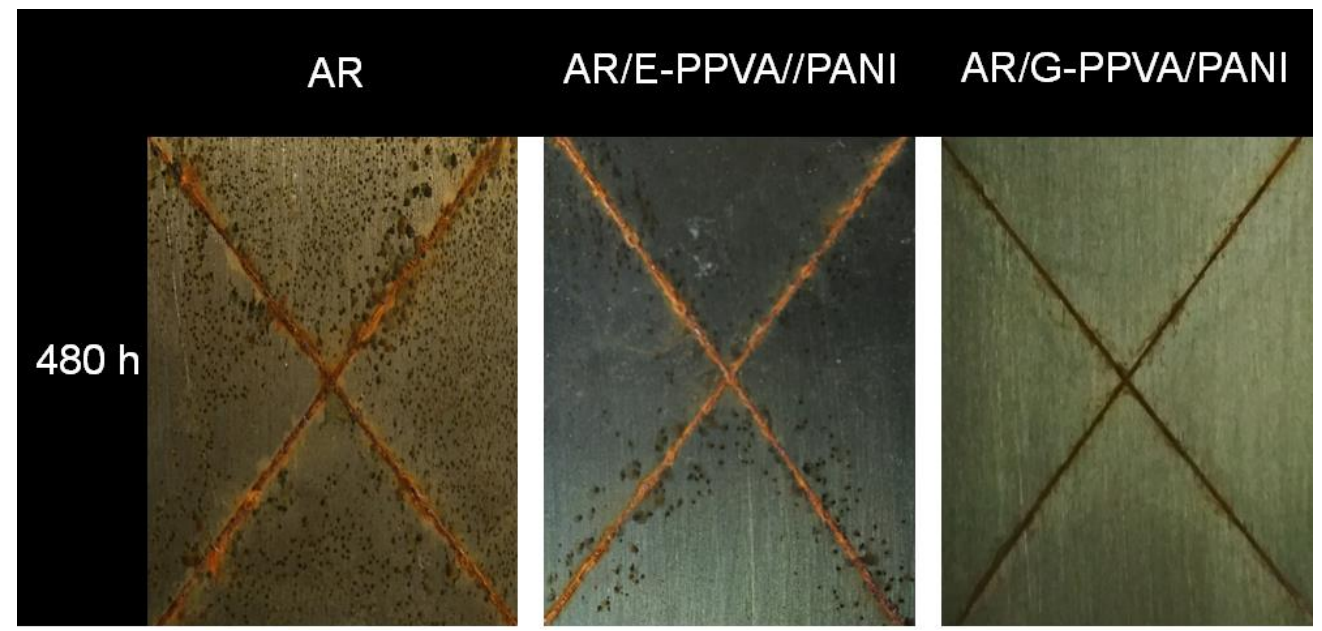

Figure 11. Optical images of coated tinplates subjected to the salt spray test for $480 \mathrm{~h}$.

Subsequently, salt spray test was utilized to macroscopically estimate the corrosion resistance, as shown in Figure 11. After $480 \mathrm{~h}$ immersion, severe corrosion diffusion and blisters were detected for AR coated tinplate. As to AR/E-PPVA/PANI coated tinplate, slight corrosion diffusion along the cut part was detected. In contrast, the corrosion was greatly weakened for AR/G-PPVA/PANI coated tinplate, and no corrosion diffusion was detected. Therefore, it can be concluded that AR/GPPVA/PANI coating was endowed with the optimum anticorrosion properties. Finally, the coating performance including hardness, adhesion strength and impact resistance was investigated, as listed in Table 2. AR/G-PPVA/PANI coating also displayed higher hardness, adhesion strength and impact resistance.

Table 2. Performance parameters of AR, AR/E-PPVA/PANI and AR/GPPVA/PANI anticorrosive coatings

\begin{tabular}{clll}
\hline Sample & Hardness & Adhesion strength & Impact resistance \\
\hline
\end{tabular}




\begin{tabular}{cccc}
\hline & & $(\mathrm{MPa})$ & $(\mathrm{Kg} . \mathrm{cm})$ \\
\hline AR & $\mathrm{H}$ & 5.32 & 50 \\
AR/E-PPVA/PANI & $2 \mathrm{H}$ & 5.37 & 55 \\
AR/G-PPVA/PANI & $2 \mathrm{H}$ & 5.68 & 55 \\
\hline
\end{tabular}

\section{Concluding remarks}

Waterborne and ecofriendly alkyd/polyaniline nanocomposite coating has been developed through incorporating polyaniline grafted polyvinyl alcohol into waterborne alkyd resin. Phosphorylated polyvinyl alcohol (PPVA) grafted with $\gamma$-glycidyl methacrylate (G-PPVA) and PPVA grafted with epichlorohydrin (E-PPVA) have been utilized to fabricate PANI grafted G-PPVA and E-PPVA aqueous dispersions. The compatibility and interaction between G-PPVA and PANI have been improved as demonstrated by the improved stability and homogeneity. G-PPVA/PANI dispersions also display good compatibility with waterborne alkyd resin. The AR/G-PPVA/PANI nanocomposite coating is endowed with superior conductivity, thermal stability and long-term corrosion resistance. The comparison of AR/G-PPVA/PANI with previous reported waterborne modified alkyd coating suggests that AR/G-PPVA/PANI has promising application as an ecofriendly, VOC-free anticorrosion coating. The developed G-PPVA/PANI may also be applied as anticorrosive additive in other waterborne polymer coatings.

\section{Notes}

The authors declare no competing financial interest.

\section{Acknowledgements}

The authors express sincere thanks to Key Research and Development Program of Shaanxi Province (No. 2018KW-007, 2017GY154), Innovation Supporting Plan of Shaanxi Province-Innovation Research Team (No. 2018TD-015), National Natural Science Foundation of China (No. 51373091), Australian National Fabrication Facility- 
Material Node (ANFF), Scientific Research Foundation (SRF) for Returned Overseas

Chinese scholars (ROCS), State Education Ministry (SEM) (No. [2012]1707).

\section{References}

[1] H. H. Wang, S. Qin, X. F. Yang, G. Q. Fei, M. Tian, Y. M. Shao, K. Zhu, A waterborne uniform graphene-poly(urethane-acrylate) complex with enhanced anticorrosive properties enabled by ionic interaction. Chem. Eng. J, 351 (2018) 939951.

[2] T. Yang, Y. Cuia, Z. Li, H. Zeng, S. Luo, W. Li, Enhancement of the corrosion resistance of epoxy coating by highly stable 3, 4, 9, 10-perylene tetracarboxylic acid functionalized grapheme, J. Hazard. Mater. 357 (2018) 475-482.

[3]S. Pathan, S. Ahmad, Green and sustainable anticorrosive coating derived from waterborne linseed alkyd using organic-inorganic hybrid cross linker, Prog. Org. Coat. 122 (2018) 189-198.

[4] J. Li, L. Ecco, M. Fedel, V. Ermini, G. Delmas, J. Pan, In-situ, AFM and EIS study of a solventborne alkyd coating with nanoclay for corrosion protection of carbon steel, Prog. Org. Coat. 87 (2015) 179-188.

[5] S. Zhong, J. Li, L. Yi, Y. Cai, W. Zhou. Cross-linked waterborne alkyd hybrid resin coatings modified by fluorinated acrylate-siloxane with high waterproof and anticorrosive performance. Polym. Adv. Tech. 30 (2019) 292-303

[6] M. Martí, G. Fabregat, D. S. Azambuja, C. Alemán, E. Armelin, Evaluation of an environmentally friendly anticorrosive pigment for alkyd primer, Prog. Org. Coat. 73 (2012) 321-329.

[7] O. u. Rahman, S. I. Bhat, H. Yu, S. Ahmad, Hyperbranched soya alkyd nanocomposites: A sustainable feedstock-based anticorrosive nanocomposite coatings, ACS Sustain. Chem. Eng. 5 (2017) 9725-9734.

[8] M. Elrebii, A.B. Mabrouk, S. Boufi, Synthesis and properties of hybrid alkydacrylic dispersions and their use in VOC-free waterborne coatings, Prog. Org. Coat. 77 (2014) 757-764.

[9] M.J. Gimeno, M. Puig, S. Chamorro, J. Molina, R. March, E. Oró, P. Pérez, J.J Gracenea, J.J. Suay, Improvement of the anticorrosive properties of an alkyd coating with zinc phosphate pigments assessed by NSS and ACET, Prog. Org. Coat. 95 (2016) 
46-53.

[10] M. Yeganeh, M. Saremi, Corrosion inhibition of magnesium using biocompatible alkyd coatings incorporated by mesoporous silica nanocontainers, Prog. Org. Coat. 79 (2015) 25-30.

[11] M.A. Deyab, Effect of carbon nano-tubes on the corrosion resistance of alkyd coating immersed in sodium chloride solution, Prog. Org. Coat. 85 (2015) 146-150.

[12] A. A. Farag, K. I. Kabel, E. M. Elnaggar, A. G. Al-Gamal, Influence of polyaniline/multiwalled carbon nanotube composites on alkyd coatings against the corrosion of carbon steel alloy. Corros. Rev. 35 (2017) 85-94

[13] M. A. Deyab, G. Mele, A. M. Al-Sabagh, E. Bloise, D. Lomonaco, S. E. Mazzetto, D.S.C. Claudenilson, Synthesis and characteristics of alkyd resin/M-Porphyrins nanocomposite for corrosion protection application, Prog. Org. Coat. 105 (2017) 286290.

[14] K. G. Chathuranga Senarathna, M.M.M.G.P.G. Mantilaka, T. A. Nirmal Peiris, H.M.T.G.A. Pitawala, D.G.G.P. Karunaratne, R.M.G.Rajapakse. Convenient routes to synthesize uncommon vaterite nanoparticles and the nanocomposites of alkyd resin/polyaniline/vaterite: The latter processing superior anticorrosive performance on mild steel surfaces. Electrochimica Acta, 117 (2014) 460-469.

[15] A. A. Salem, B. N. Grgur. Corrosion of mild steel with composite alkyd polyaniline-benzoate coating. Int. J. Electrochem Sci, 2017, 12 (2017) 8683-8694.

[16] E. Armelin, C. Ocampo, F. Liesa, J.I. Iribarren, X. Ramis, C. Alemán, Study of epoxy and alkyd coatings modified with emeraldine base form of polyaniline, Prog. Org. Coat. 58 (2007) 316-322.

[17] T. Gurunathan, C.R.K. Rao, R. Narayan, K.V.S.N. Raju, Synthesis, characterization and corrosion evaluation on new cationomeric polyurethane water dispersions and their polyaniline composites, Prog. Org. Coat. 76 (2013) 639-647.

[18] S. Pathan, S. Ahmad, s-Triazine ring-modified waterborne alkyd: synthesis, characterization, antibacterial, and electrochemical corrosion Studies, ACS. Sustain. Chem. Eng. 1 (2013), 1246-1257.

[19] S. K. Dhoke, A. S. Khanna, T. J. M. Sinha, Effect of nano-ZnO particles on the corrosion behavior of alkyd-based waterborne coatings, Prog. Org. Coat. 64 (2009) 371-382.

[20] S. Qiu, C. Chen, M. Cui, et al. Corrosion Protection Performance of Waterborne 
Epoxy Coatings Containing Self-Doped Polyaniline Nanofiber, Appl. Surf. Sci. 407 (2017) 213-222.

[21] A. Olad, M. Barati, S. Behboudi, Preparation of PANI/epoxy/Zn nanocomposite using $\mathrm{Zn}$ nanoparticles and epoxy resin as additives and investigation of its corrosion protection behavior on iron, Prog. Org. Coat. 74 (2012) 221-227.

[22] Q. Wang, L. Shao, Z. L. Ma, J. J. Xu, Y. Li, C. Y. Wang, Hierarchical porous PANI/MIL-101 nanocomposites based solid-state flexible supercapacitor, Electrochim. Acta. 281 (2018) 582-593.

[23] J. Alam, U. Riaz, S Ahmad, High performance corrosion resistant polyaniline/alkyd ecofriendly coatings, Curr. Appl. Phys. 9 (2009) 80-86.

[24] G.S. Gonçalves, A.F. Baldissera, L.F. Rodrigues Jr, E.M.A. Martini, C. A. Ferreira, Alkyd coatings containing polyanilines for corrosion protection of mild steel, Synth. Met. 161 (2011) 313-323.

[25] L.G.Ecco, M. Fedel, A. Ahniyaz, F. Deflorian, Influence of polyaniline and cerium oxide nanoparticles on the corrosion protection properties of alkyd coating, Prog. Org. Coat. 77 (2014) 2031-2038.

[26] K.G.C. Senarathna, M.M.M.G.P.G. Mantilaka, T.A.N. Peiris, H.M.T.G.A. Pitawala, D.G.G.P. Karunaratne, R.M.G. Rajapakse, Convenient routes to synthesize uncommon vaterite nanoparticles and the nanocomposites of alkyd resin/polyaniline/vaterite: The latter possessing superior anticorrosive performance on mild steel surfaces, Electrochim. Acta. 117 (2014) 460-469.

[27] H. H. Wang, R. Y. Guo, Y. D. Shen, Y. M. Shao, G. Q. Fei, K. Zhu, Waterborne polyaniline-graft-alkyd for anticorrosion coating and comparison study with physical blend. Prog. Org. Coat. 126 (2019) 187-195.

[28] F. Chen, P. Liu. Conducting polyaniline nanoparticles and their dispersion for waterborne corrosion protection coating, ACS Appl. Mat. Interfaces. 3 (2011) 26942702.

[29] X. Z. Gao, H.J. Liu, F. Cheng, Y. Chen, Thermoresponsive polyaniline nanoparticles: Preparation, characterization, and their potential application in waterborne anticorrosion coatings, Chem. Eng. J. 283 (2016) 682-691.

[30] P. Varakirkkulchai, S. Kongparakul, P. Prasassarakich, Polyaniline/polyacrylate 
core-shell composites: Preparation, morphology and anticorrosive properties, Prog. Org. Coat. 85 (2015) 84-91.

[31] H.S.O. Chan, P.K.H. Ho, S.C. Ng, B.T.G. Tan, K.L.Tan, A new water-soluble, selfdoping conducting polyaniline from poly(o-aminobenzylphosphonic acid) and its sodium salts: synthesis and characterization, J. Am. Chem. Soc. 117 (1995) 8517-8523. [32] S.A. Chen, G.W. Hwang, Water-soluble self-acid-doped conducting polyaniline: structure and properties, J. Am. Chem. Soc. 117 (1995) 10055-10062.

[33] R. Cruz-Silva, C. Ruiz-Flores, L. Arizmendi, J. Romero-García, E. Arias-Marin, I. Moggio, F.F. Castillon, M.H. Farias, Enzymatic synthesis of colloidal polyaniline particles, Polymer. 47 (2006) 1563-1568

[34] P. Ghosh, A. Chakrabarti, S.K. Siddhanta, Studies on stable aqueous polyaniline prepared with the use of polyacrylamide as the water soluble support polymer, Eur. Polym. J. 35 (1999) 803-813.

[35] C. Dispenza, M.A. Sabatino, D. Chmielewska, C. Lopresti, G. Battaglia, Inherently fluorescent polyaniline nanoparticles in a dynamic landscape, React. Funct. Polym. 72 (2012) 185-197.

[36] J. Luo, H.M. Zhang, X.H. Wang, J. Li, F.S. Wang, Stable aqueous dispersion of conducting polyaniline with high electrical conductivity, Macromolecules. 40 (2007) 8132-8135.

[37] H. M. Zhang, J.L. Lu, X.H. Wang, J. Li, S. F. Wang, From amorphous to crystalline: Practical way to improve electrical conductivity of water-borne conducting polyaniline, Polymer. 52 (2011) 3059-3064.

[38] S. K. Dhoke, A. S. Khanna, Effect of nano-Fe2O3 particles on the corrosion behavior of alkyd based waterborne coatings, Corros. Sci. 51 (2009) 6-20.

[39] H. H. Wang, H. Wen, B. Hu, G.Q. Fei, Y. D. Shen, L. Y. Sun, D. Yang, Facile approach to fabricate waterborne polyaniline nanocomposites with environmental benignity and high physical properties, Sci. Rep. 7 (2017) 43694.

[40] A. Rose, K G. Prasad, T. Sakthivel, et al. Electrochemical Analysis of Graphene Oxide/Polyaniline/Polyvinyl Alcohol Composite Nanofibers for Supercapacitor Applications, Appl. Surf. Sci. 449 (2018).

[41] H. H. Wang, X. Qin, G.Q. Fei, M. Tian, H. Wen, K. Zhu, Optimization of stability and properties of waterborne polyaniline-graft-poly(vinyl alcohol) nanocomposites 
with controllable content, Colloid. Polym. Sci. 296 (2018) 585-594.

[42] F. Chen, P. Liu, Conducting polyaniline nanoparticles and their dispersion for waterborne corrosion protection coatings, ACS Appl. Mater. Interfaces. 3 (2011) 26942702.

[43] H. H. Wang, Y. K. Niu, G.Q. Fei, Y. D. Shen, J. Lan ,In-situ polymerization, rheology, morphology and properties of stable alkoxysilane-functionalized poly(urethane-acrylate) microemulsion, Prog. Org. Coat. 99 (2016) 400-411.

[44]F. Chen, P. Liu, Highly electrically conductive polyaniline/partially phosphorylated poly(vinyl alcohol) composite films via aqueous dispersions, Macromol. Res. 19 (2011) 883-890.

[45] E. C. Venancio, P. C. Wang, A. G. MacDiarmid, The azanes: a class of material incorporating nano/micro self-assembled hollow spheres obtained by aqueous oxidative polymerization of aniline, Synth. Met. 156 (5-6) (2006) 357-369.

[46] E. S. Lopes, E. Domingos, R. S. Neves, W. Romão, K. R. de Souza, R. Valaski, B. S. Archanjo, F. G. Souza Jr., A. M. Silva, A. Kuznetsov, J. R. Araujo, The role of intermolecular interactions in polyaniline/polyamide-6,6 pressure-sensitive blends studied by DFT and ${ }^{1}$ H NMR. Eur. Polym. J. 85 (2016) 588-604.

[47] H. H. Wang , L.Y. Sun, G.Q. Fei , J. Fan, X. Y. Shi, A facile approach to fabricate waterborne, nanosized polyaniline-graft-(sulfonated polyurethane) as environmental antistatic coating. J. Appl. Polym. Sci. 134 (2017) 41-45412.

[48] P. A. Kilmartin, M. Gizdavic-Nikolaidis, Z. Zujovic, J. Travas-Sejdic, G. A. Bowmaker, R. P. Cooney, Free radical scavenging and antioxidant properties of conducting polymers examined using EPR and NMR spectroscopies. Synth. Met. 153 (2005) 153-156.

[49] J. Bhadra, N.K. Madi, N.J. Al-Thani, M.A. Al-Maadeed, Polyaniline/polyvinyl alcohol blends: Effect of sulfonic acid dopantson microstructural, optical, thermal and electrical properties, Synth. Met. 191 (2014) 126-134.

[50] J. A. Syed, S. Tang, H. Lu, X. Meng, Water-soluble polyaniline-polyacrylic acid composites as efficient corrosion inhibitors for 316ss. Ind. Eng. Chem. Res. 54(2015) 2950-2959. 